\title{
Effect of Seed Priming on Specific Activity of Acid Phosphatase and $\alpha$-Amylase in Germinating Seeds and Correlation Study in Onion (Allium cepa L.)
}

\author{
B. D. Patil*, A. R. Aher, V. R. Awari and M. R. Manjare \\ RSJRS, Kolhapur, Mahatma Phule Krishi Vidyapeeth, Rahuri, India \\ *Corresponding author
}

Keywords

Onion, Allium cepa L., Seed priming, Acid Phosphatase, $\alpha$-Amylase, Correlation Germination

Article Info

Accepted:

08 December 2020

Available Online:

10 January 2021

\section{A B S T R A C T}

An experiment was laid out in a factorial RBD with three replications involving two lots i.e. fresh and one year old onion seeds and eight seed priming treatments. The seeds were primed with zinc $(0.5$ and $0.75 \%)$, magnesium $(0.5$ and $0.75 \%)$, calcium $(0.5$ and $0.75 \%)$ and $\mathrm{GA}_{3}(50$ and $100 \mathrm{ppm})$ for 6 hours before laboratory germination and untreated control. The enzyme activity for acid phosphatase and $\alpha$-amylase at $6 \mathrm{hr}$ priming of seed, $3^{\text {rd }}$ day to germination, $6^{\text {th }}$ day to germination, $9^{\text {th }}$ day to germination and $12^{\text {th }}$ day to germination was carried out in laboratory. All the treatments in fresh seed lot showed higher acid phosphatase activity upto $3^{\text {rd }}$ day of germination than old seed lot. However, old seed lot treatments surpassed fresh seed lot treatments on $6^{\text {th }}$ day analysis. Positive and non significant correlation up to 6 days of germination was observed in old seed lot for germination. In the case of fresh seed lot positive and non significant correlation was observed for germination. In the present investigation $\alpha$-amylase activity in old untreated seed was more (0.90) as compared to fresh untreated seed (0.34). After $6 \mathrm{~h}$ seed priming $\alpha$-amylase activity was more in old seed lot treatments than fresh seed lot treatments. In the case of old seed lot treatments $\alpha$-amylase activity increased upto $6^{\text {th }}$ day except in old untreated seed wherein it increased upto $9^{\text {th }}$ day. In the case of old seed lot positive significant correlation for $\alpha$ - amylase up to 6 days of germination was found. In fresh seed lot significant correlation for $\alpha$-amylase was found except at 9 days to germination.

\section{Introduction}

The onion seed being small in size requires due cares for sowing in nursery. The unavailability/ scanty supply of fresh seed, force farmers to use aged seed for sowing in nursery of which germination is low because of decline in viability. The establishment of seedling is an important factor in bulb production and largely depends on the seed germination and vigour. The pre-sowing seed treatment has been demonstrated to give better seedling under stress conditions like very high or low temperature. To overcome the situation, seed priming is the best option. It is controlled hydration process followed by redrying that permits all metabolic processes but prevent radicle protrusion. Seed priming 
appears to reverse the detrimental effects of seed deterioration. During priming repair of DNA, RNA, protein, membranes and enzymes occurs. Oxygen is also increased, suggesting that respiratory activity is an essential component of repair. Priming increases enzyme activity such as $\alpha$ - amylase activity which breakdown starch stored in seeds to be utilized by growing embryos during germination. Acid phosphatase is believed to be important for phosphorus scavenging and remobilization in plants.

Enzyme helps for many physiological processes, including regulation of phosphorus efficiency. $\alpha$ - amylase plays an important role in hydrolyzing the endosperm starch into sugars, which provide the energy for the growth of roots and shoots (Kaneko et al., 2002). $\alpha$-amylase hydrolyzes $\alpha-1,4$ linkage of starch in a random manner playing an important role in starch metabolism in germinating seed.

\section{Materials and Methods}

The specific activity of $\alpha$-amylase and acid phosphatase enzymes after priming of seed for 6 hours, 3 day old sprouted seed, 6 day old seedling, 9 day old seedling and 12 day old seedling was carried out in laboratory as per the standard methods of Biochemical Analysis (Thimmaiah, 2009).

100 seeds in four replications from each treatment were kept for germination at $20^{\mathrm{O}} \mathrm{C}$ temperature for 12 days. The seedlings were categorized into normal, abnormal seedlings and dead seeds. Accordingly germination percentage was computed on normal seedling. (Anonymous, 1985).

\section{Results and Discussion}

Activities of several enzymes associated with the germination process have been proven to change in response to seed priming. These include increases in the activities of acid phosphatase and esterase in lettuce (Khan et al., 1978), $\alpha$-amylase in rice (Farooq et al., 2006) and antioxidant enzymes in wheat (Afzal et al., 2006). Several enzymes are activated during seed germination including acid phosphatase implicated in the remobilization of phosphorus reserves (Biswas and Cundiff, 1991). Acid phosphatase is believed to be important for phosphorus scavenging and remobilization in plants, also plays important role in many physiological processes, including regulation of phosphorus efficiency.

As per table. 1 and Fig 1 the acid phosphatase activity was found to be higher in fresh untreated seed $(0.107)$ than old untreated seed (0.088). All the treatments in fresh seed lot showed higher activity upto $3^{\text {rd }}$ day of germination than old seed lot. However old seed lot treatments surpassed fresh seed lot treatments on $6^{\text {th }}$ day analysis. In old seed lot treatments the activity increased upto $9^{\text {th }}$ day except in seed priming treatment with $\mathrm{Mg}$ $0.75 \%$. In fresh seed lot treatments the activity increased in $\mathrm{Zn} 0.5 \%$, Ca $0.75 \%$, GA $50 \mathrm{ppm}$ and untreated fresh seed upto $9^{\text {th }}$ day. In rest of the treatments of fresh lot, the activity showed increased trend upto $12^{\text {th }}$ day except in GA 100 ppm in which the activity was same on both days.

The stimulation of phosphatases activities appears to maintain higher cell metabolic status by providing a higher rate of phosphate release and active transport and biosynthetic events in growing embryo axes (Dubey and Sharma, 1990). Nasri et al., (2011) showed that there was significant improvement in acid phosphatase and phytase activities in roots, shoots and cotyledons due to osmopriming treatment. These enzymes activities were greater in primed seeds than in non-primed seeds in saline conditions. 
Table.1 Effect of seed lots and seed priming treatments on specific activity for acid phosphatase ( $\mu$ moles of p-nitrophenol formed $\min ^{-1} \mathrm{mg}^{-1}$ protein) of six hour primed seed, 3 day germinated seed, 6 day and 9 day old seedling

\begin{tabular}{|c|c|c|c|c|c|c|c|c|c|c|c|c|c|c|c|}
\hline \multirow{3}{*}{ Treatments } & \multicolumn{3}{|c|}{ Sp. act of $6 \mathrm{hr}$ primed seeds } & \multicolumn{3}{|c|}{$\begin{array}{c}\text { Sp. act of } 3 \text { day germinated } \\
\text { seed }\end{array}$} & \multicolumn{3}{|c|}{ Sp. act of 6 day old seedling } & \multicolumn{3}{|c|}{ Sp. act of 9 day old seedling } & \multicolumn{3}{|c|}{ Sp. act of 12 day germinated seed } \\
\hline & \multicolumn{2}{|c|}{ Seed Lot } & \multirow[t]{2}{*}{ Mean } & \multicolumn{2}{|c|}{ Seed Lot } & \multirow[t]{2}{*}{ Mean } & \multicolumn{2}{|c|}{ Seed Lot } & \multirow[t]{2}{*}{ Mean } & \multicolumn{2}{|c|}{ Seed Lot } & \multirow[t]{2}{*}{ Mean } & \multicolumn{2}{|c|}{ Seed Lot } & \multirow{2}{*}{ Mean } \\
\hline & $\begin{array}{c}\mathbf{L}_{1} \\
(\text { Old })\end{array}$ & $\begin{array}{c}\mathbf{L}_{2} \\
\text { (Fresh) }\end{array}$ & & $\begin{array}{c}\mathbf{L}_{1} \\
(\text { Old })\end{array}$ & $\begin{array}{c}\mathbf{L}_{2} \\
\text { (Fresh) }\end{array}$ & & $\begin{array}{c}\mathbf{L}_{1} \\
(\text { Old })\end{array}$ & $\begin{array}{c}\mathbf{L}_{2} \\
\text { (Fresh) }\end{array}$ & & $\begin{array}{c}\mathbf{L}_{1} \\
(\text { Old })\end{array}$ & $\begin{array}{c}\mathbf{L}_{2} \\
(\text { Fresh) }\end{array}$ & & $\begin{array}{c}\mathbf{L}_{1} \\
(\text { Old })\end{array}$ & $\begin{array}{c}\mathbf{L}_{2} \\
\text { (Fresh) }\end{array}$ & \\
\hline$T_{1}($ Zinc- $0.5 \%)$ & 0.101 & 0.112 & 0.107 & 0.171 & 0.218 & 0.194 & 0.80 & 0.74 & 0.77 & 0.86 & 0.91 & 0.89 & 0.83 & 0.82 & 0.83 \\
\hline $\mathrm{T}_{2}($ Zinc $-0.75 \%)$ & 0.094 & 0.120 & 0.107 & 0.172 & 0.235 & 0.203 & 0.83 & 0.73 & 0.78 & 0.85 & 0.88 & 0.86 & 0.79 & 0.89 & 0.84 \\
\hline $\mathrm{T}_{3}(\mathrm{Mg}-0.5 \%)$ & 0.098 & 0.118 & 0.108 & 0.181 & 0.228 & 0.205 & 0.77 & 0.73 & 0.75 & 0.83 & 0.83 & 0.83 & 0.81 & 0.91 & 0.86 \\
\hline $\mathrm{T}_{4}(\mathrm{Mg}$-. $75 \%)$ & 0.114 & 0.124 & 0.119 & 0.188 & 0.220 & 0.204 & 0.77 & 0.72 & 0.74 & 0.82 & 0.82 & 0.82 & 0.86 & 0.84 & 0.85 \\
\hline $\mathrm{T}_{5}(\mathrm{Ca}-0.5 \%)$ & 0.107 & 0.128 & 0.118 & 0.177 & 0.210 & 0.194 & 0.81 & 0.70 & 0.75 & 0.87 & 0.80 & 0.83 & 0.84 & 0.85 & 0.84 \\
\hline $\mathrm{T}_{6}(\mathrm{Ca}-0.75 \%)$ & 0.129 & 0.132 & 0.131 & 0.197 & 0.212 & 0.205 & 0.79 & 0.71 & 0.75 & 0.87 & 0.83 & 0.85 & 0.80 & 0.76 & 0.78 \\
\hline $\mathrm{T}_{7}\left(\mathrm{GA}_{3}-\mathbf{5 0} \mathbf{p p m}\right)$ & 0.128 & 0.121 & 0.124 & 0.187 & 0.211 & 0.199 & 0.82 & 0.72 & 0.77 & 0.91 & 0.84 & 0.87 & 0.79 & 0.79 & 0.79 \\
\hline $\begin{array}{l}\mathrm{T}_{8}\left(\mathrm{GA}_{3}-100\right. \\
\mathrm{ppm})\end{array}$ & 0.139 & 0.138 & 0.138 & 0.181 & 0.216 & 0.199 & 0.86 & 0.70 & 0.78 & 0.89 & 0.86 & 0.87 & 0.76 & 0.86 & 0.81 \\
\hline $\mathbf{T}_{9}$ (Control) & 0.088 & 0.107 & 0.100 & 0.179 & 0.201 & 0.190 & 0.63 & 0.58 & 0.60 & 0.91 & 0.86 & 0.88 & 0.89 & 0.75 & 0.82 \\
\hline Mean & 0.111 & 0.122 & 0.097 & 0.181 & 0.217 & 0.199 & 0.79 & 0.70 & 0.75 & 0.87 & 0.85 & 0.86 & 0.82 & 0.83 & 0.82 \\
\hline \multirow[t]{2}{*}{ S. E. \pm} & Lot & Treat & LxT & Lot & Treat & LxT & Lot & Treat & LxT & Lot & Treat & LxT & Lot & Treat & LxT \\
\hline & 0.0004 & 0.0008 & 0.0012 & 0.0004 & 0.0009 & 0.0013 & 0.0031 & 0.0066 & 0.0093 & 0.0041 & 0.0088 & 0.0124 & 0.004 & 0.0085 & 0.012 \\
\hline C. D. & 0.0011 & 0.0023 & 0.0032 & 0.0012 & 0.0026 & 0.0037 & 0.0089 & 0.0189 & 0.0268 & 0.012 & 0.0252 & 0.0357 & 0.0115 & 0.0245 & 0.0346 \\
\hline
\end{tabular}


Table. 2 Effect of seed lots and seed priming treatments on specific activity for $\alpha$-amylase ( $\mu \mathrm{g}$ of maltose released min $\mathrm{mg}^{-1} \mathrm{protein}$ ) of $6 \mathrm{hr}$ primed seed, 3 day germinated seed, 6 day and 9 day old seedling

\begin{tabular}{|c|c|c|c|c|c|c|c|c|c|c|c|c|c|c|c|}
\hline \multirow{3}{*}{ Treatments } & \multicolumn{3}{|c|}{ Sp. act of $6 \mathrm{hr}$ primed seeds } & \multicolumn{3}{|c|}{$\begin{array}{l}\text { Sp. act of } 3 \text { day germinated } \\
\text { seed }\end{array}$} & \multicolumn{3}{|c|}{ Sp. act of 6 day old seedling } & \multicolumn{3}{|c|}{ Sp. act of 9 day old seedling } & \multicolumn{3}{|c|}{$\begin{array}{c}\begin{array}{c}\text { Sp. act of } 12 \text { day germinated } \\
\text { seed }\end{array} \\
\text {. }\end{array}$} \\
\hline & \multicolumn{2}{|c|}{ Seed Lot } & \multirow[t]{2}{*}{ Mean } & \multicolumn{2}{|c|}{ Seed Lot } & \multirow[t]{2}{*}{ Mean } & \multicolumn{2}{|c|}{ Seed Lot } & \multirow[t]{2}{*}{ Mean } & \multicolumn{2}{|c|}{ Seed Lot } & \multirow[t]{2}{*}{ Mean } & \multicolumn{2}{|c|}{ Seed Lot } & \multirow{2}{*}{ Mean } \\
\hline & $\begin{array}{c}\mathrm{L}_{1} \\
(\text { Old })\end{array}$ & $\begin{array}{c}\mathbf{L}_{2} \\
(\text { Fresh })\end{array}$ & & $\begin{array}{c}\mathbf{L}_{1} \\
(\text { Old })\end{array}$ & $\begin{array}{c}\mathbf{L}_{2} \\
(\text { Fresh) }\end{array}$ & & $\begin{array}{c}\mathbf{L}_{1} \\
(\text { Old })\end{array}$ & $\begin{array}{c}\mathbf{L}_{2} \\
\text { (Fresh) }\end{array}$ & & $\begin{array}{c}\mathbf{L}_{1} \\
(\text { Old })\end{array}$ & $\begin{array}{c}\mathbf{L}_{2} \\
(\text { Fresh })\end{array}$ & & $\begin{array}{c}\mathbf{L}_{1} \\
(\text { Old })\end{array}$ & $\begin{array}{c}\mathbf{L}_{2} \\
(\text { Fresh) }\end{array}$ & \\
\hline$T_{1}($ Zinc- $0.5 \%)$ & 1.27 & 1.04 & 1.15 & 7.28 & 5.49 & 6.38 & 9.70 & 7.37 & 8.53 & 9.14 & 8.75 & 8.94 & 2.94 & 3.83 & 3.38 \\
\hline $\mathrm{T}_{2}($ Zinc $-0.75 \%)$ & 1.00 & 0.88 & 0.94 & 7.08 & 5.46 & 6.27 & 9.57 & 7.27 & 8.42 & 9.08 & 8.63 & 8.85 & 3.21 & 3.05 & 3.13 \\
\hline $\mathrm{T}_{3}(\mathrm{Mg}-0.5 \%)$ & 1.10 & 0.87 & 0.98 & 7.70 & 5.37 & 6.56 & 11.28 & 7.88 & 9.58 & 10.05 & 8.47 & 9.26 & 3.07 & 3.48 & 3.27 \\
\hline $\mathrm{T}_{4}(\mathrm{Mg}-.75 \%)$ & 1.31 & 1.14 & 1.22 & 7.65 & 5.31 & 6.50 & 10.89 & 7.95 & 9.42 & 9.89 & 8.51 & 9.20 & 3.38 & 3.77 & 3.57 \\
\hline $\mathrm{T}_{5}(\mathrm{Ca}-0.5 \%)$ & 1.38 & 1.06 & 1.22 & 7.14 & 5.37 & 6.25 & 10.83 & 8.01 & 9.42 & 9.67 & 8.85 & 9.26 & 3.16 & 4.44 & 3.80 \\
\hline $\mathrm{T}_{6}(\mathrm{Ca}-0.75 \%)$ & 1.25 & 1.11 & 1.18 & 7.66 & 5.44 & 6.55 & 10.60 & 7.95 & 9.28 & 9.66 & 9.03 & 9.34 & 3.53 & 4.38 & 3.96 \\
\hline $\mathrm{T}_{7}\left(\mathrm{GA}_{3}-50 \mathrm{ppm}\right)$ & 1.25 & 1.09 & 1.17 & 7.58 & 5.36 & 6.47 & 9.92 & 8.21 & 9.06 & 9.38 & 8.74 & 9.06 & 3.64 & 4.64 & 4.14 \\
\hline$T_{8}\left(G_{3}-100 p p m\right)$ & 1.21 & 1.04 & 1.13 & 7.85 & 5.39 & 6.62 & 10.37 & 8.11 & 9.24 & 9.06 & 8.52 & 8.79 & 3.89 & 4.42 & 4.15 \\
\hline $\mathbf{T}_{9}$ (Control) & 0.90 & 0.34 & 0.62 & 4.91 & 4.37 & 4.64 & 6.18 & 6.95 & 6.57 & 9.77 & 8.43 & 9.10 & 4.66 & 3.12 & 3.89 \\
\hline Mean & 1.19 & 0.95 & 1.07 & 7.23 & 5.28 & 6.25 & 9.93 & 7.74 & 8.84 & 9.52 & 8.66 & 9.09 & 3.50 & 3.90 & 3.70 \\
\hline \multirow[t]{2}{*}{ S. E. \pm} & Lot & Treat & LxT & Lot & Treat & LxT & Lot & Treat & LxT & Lot & Treat & LxT & Lot & Treat & LxT \\
\hline & 0.005 & 0.0108 & 0.0152 & 0.0267 & 0.0567 & 0.0802 & 0.0171 & 0.0364 & 0.0514 & 0.0109 & 0.0233 & 0.033 & 0.0109 & 0.023 & 0.0326 \\
\hline C. D. & 0.0145 & 0.0309 & 0.0438 & 0.0768 & 0.1629 & 0.2304 & 0.0493 & 0.105 & 0.1478 & 0.0316 & 0.067 & 0.0948 & 0.0312 & 0.0662 & 0.0934 \\
\hline
\end{tabular}


Table.3 Initial and final germination (\%), seedling vigour index and field emergence (\%) as influenced by seed lots and seed priming treatments

\begin{tabular}{|c|c|c|c|c|c|c|c|c|c|c|c|c|}
\hline \multirow[t]{3}{*}{ Treatments } & \multicolumn{3}{|c|}{$\begin{array}{l}\text { Germination (first } \\
\text { count) on } 6^{\text {th }} \text { day }(\%)\end{array}$} & \multicolumn{3}{|c|}{$\begin{array}{c}\text { Final germination on } 12^{\text {th }} \\
\text { day }(\%)\end{array}$} & \multicolumn{3}{|c|}{ Seedling vigour index } & \multicolumn{3}{|c|}{ Field emergence } \\
\hline & \multicolumn{2}{|c|}{ Seed lot } & \multirow{2}{*}{$\begin{array}{c}\text { Mea } \\
\mathbf{n}\end{array}$} & \multicolumn{2}{|c|}{ Seed lot } & \multirow[t]{2}{*}{ Mean } & \multicolumn{2}{|c|}{ Seed lot } & \multirow[t]{2}{*}{ Mean } & \multicolumn{2}{|c|}{ Seed lot } & \multirow[t]{2}{*}{ Mean } \\
\hline & $\begin{array}{c}\mathbf{L}_{1} \\
\text { (Old) }\end{array}$ & $\begin{array}{c}\mathbf{L}_{2} \\
(\text { Fresh })\end{array}$ & & $\begin{array}{c}\mathbf{L}_{1} \\
(\mathbf{O l d})\end{array}$ & $\begin{array}{c}\mathbf{L}_{2} \\
\text { (Fresh) }\end{array}$ & & $\begin{array}{c}\mathbf{L}_{1} \\
(\mathbf{O l d})\end{array}$ & $\begin{array}{c}\mathbf{L}_{2} \\
\text { (Fresh) }\end{array}$ & & $\begin{array}{c}\mathbf{L}_{1} \\
(\mathbf{O l d})\end{array}$ & $\begin{array}{c}\mathbf{L}_{2} \\
\text { (Fresh) }\end{array}$ & \\
\hline $\mathrm{T}_{1}($ Zinc $-0.5 \%)$ & 72.67 & 78.67 & 75.67 & 80.33 & 86.67 & 83.50 & 1346.10 & 1357.40 & 1351.75 & 68.67 & 73.67 & 71.17 \\
\hline$T_{2}($ Zinc $-0.75 \%)$ & 72.33 & 79.67 & 76.00 & 80.33 & 86.00 & 83.17 & 1396.21 & 1301.72 & 1348.96 & 65.33 & 73.00 & 69.16 \\
\hline$T_{3}$ (Magnesium- $0.5 \%$ ) & 73.67 & 76.00 & 74.83 & 81.00 & 85.33 & 83.17 & 1335.62 & 1288.53 & 1312.07 & 71.67 & 76.00 & 73.83 \\
\hline $\mathbf{T}_{4}($ Magnesium- $0.75 \%)$ & 74.67 & 79.00 & 76.83 & 80.33 & 86.67 & 83.50 & 1301.77 & 1326.45 & 1314.11 & 72.00 & 73.33 & 72.67 \\
\hline $\mathbf{T}_{5}($ Calcium - $0.5 \%)$ & 71.33 & 75.33 & 73.33 & 80.67 & 86.33 & 83.50 & 1337.17 & 1327.05 & 1332.11 & 69.33 & 73.67 & 71.50 \\
\hline$T_{6}($ Calcium - 0.75\% $)$ & 70.67 & 77.67 & 74.17 & 81.33 & 85.33 & 83.33 & 1358.00 & 1304.03 & 1331.01 & 69.33 & 74.67 & 72.00 \\
\hline$T_{7}\left(G_{3}-50\right.$ ppm $)$ & 73.67 & 78.67 & 76.17 & 81.00 & 85.67 & 83.33 & 1330.42 & 1333.89 & 1332.16 & 73.00 & 74.67 & 73.83 \\
\hline$T_{8}\left(G_{3}-100\right.$ ppm $)$ & 70.67 & 78.67 & 74.67 & 81.33 & 85.67 & 83.50 & 1382.23 & 1340.04 & 1361.13 & 72.67 & 74.33 & 73.50 \\
\hline $\mathbf{T}_{9}$ (Control) & 63.33 & 76.33 & 69.83 & 77.00 & 84.33 & 80.67 & 1237.70 & 1284.00 & 1260.85 & 64.00 & 72.00 & 68.00 \\
\hline Mean & 71.44 & 77.78 & 74.61 & 80.37 & 85.78 & 83.07 & 1336.14 & 1318.12 & 1327.13 & 69.56 & 73.93 & 71.74 \\
\hline \multirow[t]{2}{*}{ S.E.土 } & Lot & Treat & LxT & Lot & Treat & LxT & Lot & Treat & LxT & Lot & Treat & LxT \\
\hline & 0.383 & 0.813 & 1.15 & 0.3155 & 0.6694 & 0.9466 & 7.301 & 15.488 & 21.903 & 0.629 & 1.334 & 1.886 \\
\hline
\end{tabular}


Table.4 Correlation of old and fresh seed lot for acid phosphatase enzyme activity with germination, seedling vigour index and field emergence

\begin{tabular}{|c|l|c|c|c|c|c|c|}
\hline $\begin{array}{c}\text { Sr. } \\
\text { No. }\end{array}$ & \multicolumn{4}{|c|}{ Old seed lot } & \multicolumn{3}{c|}{ Fresh seed lot } \\
\hline & Particular & $\begin{array}{c}\text { Germi- } \\
\text { nation. }\end{array}$ & S.V.I. & $\begin{array}{c}\text { Field } \\
\text { emergence }\end{array}$ & $\begin{array}{c}\text { Germi- } \\
\text { nation }\end{array}$ & S.V.I. & $\begin{array}{c}\text { Field } \\
\text { emergence }\end{array}$ \\
\hline 1. & 6 hr. priming & 0.619 & - & - & 0.313 & - & - \\
\hline 2. & 3 Days & 0.386 & - & - & 0.692 & - & - \\
\hline 3. & 6 Days & 0.758 & - & - & 0.509 & - & - \\
\hline 4. & 9 Days & -0.406 & -0.578 & -0.262 & 0.002 & -0.199 & -0.253 \\
\hline $\mathbf{5 .}$ & 12 Days & $-0.947 *$ & $-0.908^{*}$ & $-0.922^{*}$ & 0.324 & 0.821 & 0.313 \\
\hline
\end{tabular}

Table.5 Correlation of old and fresh seed lot for $\alpha$-amylase enzyme activity with germination, seedling vigour index and field emergence

\begin{tabular}{|l|l|l|l|l|c|c|c|}
\hline Sr. & \multicolumn{4}{|c|}{ Old seed lot } & \multicolumn{3}{c|}{ Fresh seed lot } \\
\cline { 3 - 9 } No. & \multicolumn{1}{|c|}{ Particular } & $\begin{array}{c}\text { Germi- } \\
\text { nation }\end{array}$ & S.V.I. & $\begin{array}{c}\text { Field } \\
\text { emergence }\end{array}$ & $\begin{array}{c}\text { Germi- } \\
\text { nation }\end{array}$ & S.V.I. & $\begin{array}{c}\text { Field } \\
\text { emergence }\end{array}$ \\
\hline 1. & 6 hr. priming & $0.879^{*}$ & - & - & $0.935^{* *}$ & - & - \\
\hline 2. & 3 Days & $0.904^{*}$ & - & - & $0.868^{*}$ & - & - \\
\hline 3. & 6 Days & $0.888^{*}$ & - & - & $0.896^{*}$ & - & - \\
\hline 4. & 9 Days & -0.637 & -0.663 & -0.836 & 0.533 & 0.484 & 0.192 \\
\hline 5. & 12 Days & -0.668 & -0.707 & -0.524 & $0.915^{*}$ & 0.878 & 0.874 \\
\hline
\end{tabular}

Fig.1 Acid phosphatase enzyme activity as influenced by seed lots and seed priming treatments

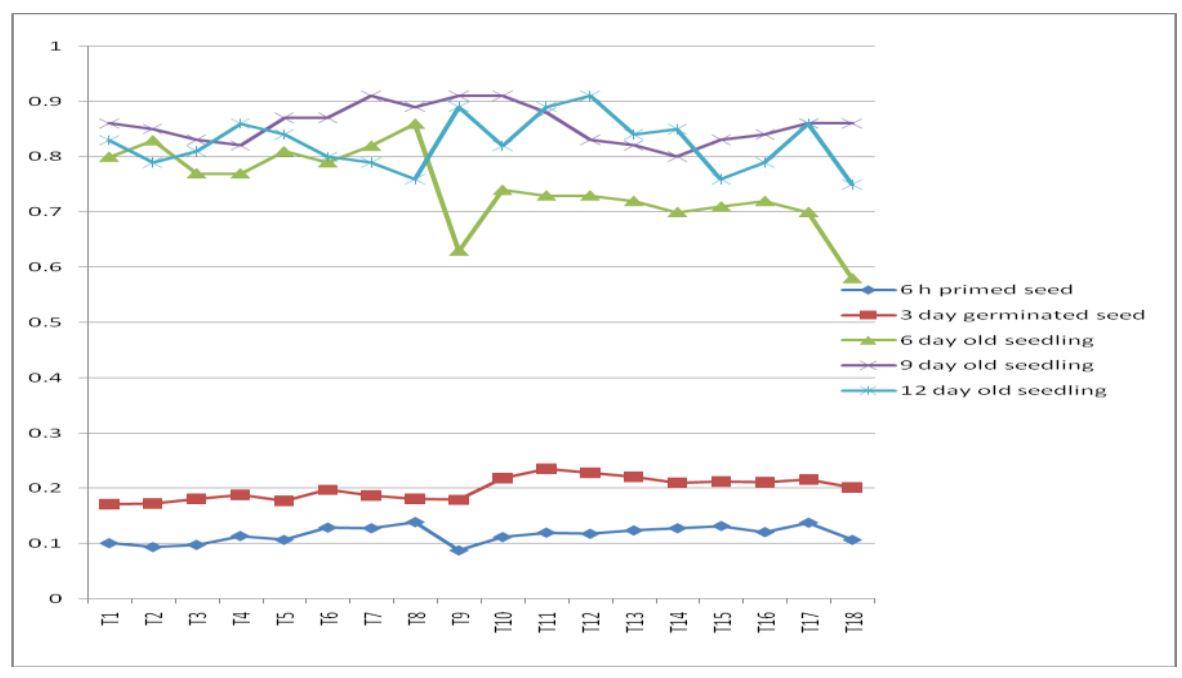


Fig.2 $\alpha$-amylase enzyme activity as influenced by seed lots and seed priming treatments

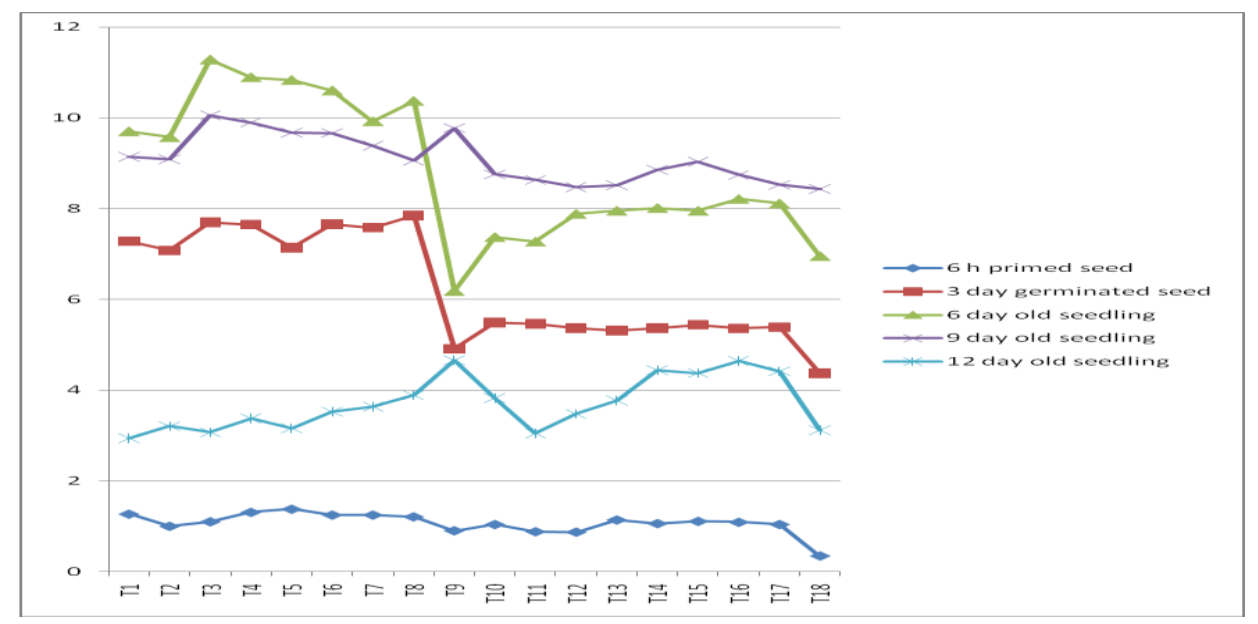

Starch is the principal storage polysaccharide in plant cells. It is made up of about $10-20 \%$ of amylose and about $80-90 \%$ of amylopectin. $\alpha$-amylase is the hydrolytic enzyme which hydrolyze starch. $\alpha$-amylase hydrolyzes $\alpha-1$, 4 linkage of starch in a random manner playing an important role in starch metabolism in germinating seed.

$\alpha$-amylase activity in old untreated seed was more $(0.90)$ as compared to fresh untreated seed (0.34). After $6 \mathrm{~h}$ seed priming $\alpha$-amylase activity was more in old seed lot treatments than fresh seed lot treatments. In the case of old seed lot treatments $\alpha$-amylase activity increased upto $6^{\text {th }}$ day except in old untreated seed wherein it increased upto $9^{\text {th }}$ day. Later on the activity decreased. $\alpha$-Amylase activity was greater in old seed lot treatments upto $9^{\text {th }}$ day as compared to fresh seed lot treatments. In old seed lot treatments, seed priming treatments with magnesium $(0.5 \%$ \& $0.75 \%)$ and calcium $(0.5 \% \quad \& \quad 0.75 \%)$ recorded comparatively higher $\alpha$-amylase activity than other treatments (Table $2 \&$ Fig 2). During the germination process, gibberellic acid is released from the embryo and activates some responsible genes of $\alpha$-amylase mRNA transcription (Taiz and Zeiger, 1998). $\alpha$ amylase plays an important role in hydrolyzing the endosperm starch into sugars, which provide the energy for the growth of roots and shoots (Kaneko et al., 2002).

Farooq et al., (2012) showed that seed priming in rice improved germination and emergence, allometry, kernel yield, and its quality. The results suggest that physiological changes produced by osmohardening enhanced the starch hydrolysis and made more sugars available for embryo growth, vigourous seedling production and, later on, improved allometric, kernel yield and quality attributes.

The data presented showed significantly better germination for seed priming with magnesium $(0.75 \%)(76.83 \%)$ at first count on $6^{\text {th }}$ day followed by $\mathrm{GA}_{3}$ (50ppm) $(76.17 \%)$. The differences in germination percentage of seeds appeared to be significantly influenced due to seed treatment (Table 3). The results of present study are in agreements with observations of Basra et al.(1989).

In the case of final germination it was observed that fresh seed lot was significantly superior than old seed lot. The seed priming treatments were non significant. The interaction was also found non-significant. 
Treatment zinc $(0.5 \%)$ and magnesium $(0.75 \%)$ showed highest germination of $86.67 \%$ while minimum germination of $77.0 \%$ was observed in control. It was noticed that due to seed priming treatments, germination of old seed lot was improved as compared to old control. Priming stimulates many of the metabolic processes involved with the early phases of germination. As part of the germination process have been initiated due to priming, seedlings from primed seed grow faster, grow more vigourously, and perform better in adverse conditions (Basker and Hatton, 1987; Desai et al., 1997).

The highest seedling vigour index of 1396.21 was observed in zinc $(0.75 \%)$ while the lowest seedling vigour index in control (1237.70). The differences for seedling vigour index were non significant for $\mathrm{L}_{1}$ and $\mathrm{L}_{2}$ seed lot, while the seed priming treatments significantly differed. The seedling vigour was highest for treatment $\mathrm{T}_{8}$ GA3 (100ppm). Corbineau et al., (2000) observed that imbibitions of tomato seeds in PEG results in sharp increases in adenosine triphosphatate (ATP), energy charge (EC) and ATP/ADP (adenosine diphosphatase) ratio. These remain higher in primed seeds even after drying than in unprimed seeds. Positive and non significant correlation up to 6 days of germination was observed in old seed lot for germination. In the case of fresh seed lot positive and non significant correlation was observed for germination.

In the case of old seed lot positive significant correlation for $\alpha$ - amylase up to 6 days of germination was found. In fresh seed lot, significant correlation were observed for $\alpha$ amylase except at 9 days to germination.

\section{References}

Afzal, I.S., M.A. Basra, A. Hameed and M. Farooq (2006). Physiological enhancements for alleviation of salt stress in wheat. Pak. J. Bot., Vol.: 38(5), pp. 1649-1659.

Anonymous (1985). International Rules for Seed Testing. Rules and Annexes. Seed Sci. and Technol. Vol.: 3(2), pp. 299513.

Baskar, A. M. and W.Hatton (1987). Calcium peroxidase as a seed coating material for paddy rice. III. Glasshouse trials. Plant Soil, Vol.: 99, pp. 379-387.

Basra, A.S., R. Dhillon and C.P.Malik (1989). Influence of seed pretreatment with plant growth regulators on metabolic alterations of germinating maize embryos under stressing temperature regimes. Ann.Bot. (London). Vol.: 64, pp. 37-41.

Biswas T.K. and C. Cundiff (1991). Multiple forms of acid phosphatase in germinating seeds of Vigna sinensis. Phytochemistry, Vol.: 30, pp.2119-2125.

Corbineau, F., N. Ozbingo, D. Vineland and D. Come (2000). Improvement of tomato seed germination by osmopriming as related to energy metabolism. In Seed Biology Advances and Applications: Proceedings of the Sixth International Workshop on Seeds (eds Black, M., K. J. Bradford and J. Vasquez-Ramos), Merida,Mexico, 1999, CABI, Cambridge, pp. 467-474.

Desai, B. B., P. M. Kotecha and D. K. Salunke (1987). Seeds Handbook, Marcel Dekker, Inc. New York.

Dubey, R. S. and K. N. Sharma (1990). Behaviour of phosphatases in germinating rice in relation to salt tolerance. Plant Physiol. Biochem.,Vol.: 28, pp.17-26.

Farooq, M., S.M.A. Basra and A. Wahid (2006). Priming of field-sown rice seed enhances germination, seedling establishment, allometry and yield. Plant Growth Regul., Vol.: 49, pp. 285-294.

Farooq, M., S.M. A. Barsa and A. Wahid 
(2012). Priming of field-sown rice seed enhances germination, seedling establishment, allometry and yield. Plant Growth Regulation. Vol.: 49(2), pp.285294.

Kaneko, M., H. Itoh, M. Ueguchi-Tanaka, M. Ashikari and M. Matsuoka (2002). The $\alpha$-amylase induction in endosperm during rice seed germination is caused by gibberellin synthesized in epithelium. Plant Physiology. Vol.: 68, pp. 12641270.

Khan, A.A., K. L. Tao, J.S. Knypl, B. Borkowska and L.E. Powell (1978). Osmotic conditioning of seeds: physiological and biochemical changes. Acta Hortic., Vol.: 83, pp. 267-282.
Nasri, N., R. Kaddour, H. Mahmoudi, O. Baatour N. Bouraoui and M. Lachaal (2011). The effect of osmopriming on germination, seedling growth and phosphatase activities of lettuce under saline condition. African Journal of Biotechnology: Vol.: 10(65) pp.1436614372.

Taiz, L. and E. Zeiger (1998). Plant Physiology.Second edition, Sinauer Association, Inc, Publishers Sunderland, Massachusetts, USA, pp.792.

Thimmaiah, S. K. (2009). Standard methods of Biochemical analysis. Kalyani Publishers. New Delhi, India. pp.94-230.

\section{How to cite this article:}

Patil, B. D., A. R. Aher, V. R. Awari and Manjare, M. R. 2021. Effect of Seed Priming on Specific Activity of Acid Phosphatase and $\alpha$-Amylase in Germinating Seeds and Correlation Study in Onion (Allium cepa L.). Int.J.Curr.Microbiol.App.Sci. 10(01): 632-640. doi: https://doi.org/10.20546/ijcmas.2021.1001.077 